\title{
e-HR
}

\section{Chat bots are the new HR managers}

\section{Beerud Sheth}

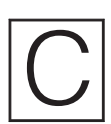

hat bots will continue to transform virtually every business function from marketing to sales to customer support. One of the other areas that we'll see a major transformation in is $\mathrm{HR}$.

HR teams have a very challenging job with seemingly conflicting objectives: keeping employees focused and working hard while also keeping them happy and satisfied. HR teams are often held accountable for employee retention and churn without the ability to manage them directly. HR is expected to have a finger on the pulse of a very large employee base while operating with a very small team. HR teams find that their responsibilities are vast and the goals are high - there's rarely enough time or resources to do justice to it all. A lot of their time is invested in managing routine processes, which impacts the focus on higher priority activities. Teams have limited time left over to offer employees the individual attention they need when handling sensitive personal issues. Even more reduced is the ability to proactively engage with employees, to guide and mentor them through their career paths.

Chat bots can help the HR professionals by dramatically amplifying their capabilities. Chat bots can help HR teams stay on top of the substantial responsibilities and achieve the impossible goals they have.

Chat bots can automate routine processes that take a lot of their time.
Recruiting activities such as screening candidates, scheduling interviews and managing the recruiting life cycle for candidates and hiring managers can be substantially automated. In fact, a startup called RoboRecruiter, built on the Gupshup platform, has developed a chat bot for automating the end-toend recruitment process, with amazing results and improvements in productivity. Employee onboarding and orientation is another area that's ripe for automation. New employees account for a high number of HR queries that can be easily automated. Even regular processes, such as attendance tracking, goal tracking, performance reviews, employee surveys, leave balances, etc. can be substantially automated. A company called QuickWork has built chat bots that are automating $\mathrm{HR}$ and related business processes for many enterprises. To be clear, even though most companies use an HR system to automate many tasks, chat bots make existing systems much more userfriendly than before, dramatically increasing the usage and compliance.

Chat bots help HR become much more accessible to employees. They can provide instant, accurate responses to common queries. The more complex queries can be automatically escalated for human review and response. Automating the frequently asked queries frees up HR teams to personally handle the more complex queries. This enables them to respond and intervene quickly in 
sensitive situations. This is especially useful during phases of change management when businesses roll out major changes in organization or strategy. These changes lead to a large number of employee queries in a very short time that have to be addressed very quickly to prevent issues from festering. The increased accessibility is particularly valuable to employees in remote locations, away from headquarters, who are deprived of the ability to have corridor conversations with HR teams.

Chat bots enable HR teams to engage with each employee on an individual, one-on-one basis, consistent with their personal situation and issues. A chat bot can maintain regular, proactive contact with each employee throughout the year to detect any issues that need to be escalated for human engagement. Tools such as natural language processing and sentiment mining can help chat bots detect anger, frustration, de-motivation, exhaustion and related issues. Based on these observations, chat bots may pull in an HR professional for human engagement. Chat bots may also proactively recommend vacation time, clubs and activities or other resources available within or outside the organization. Chat bots may optionally help employees be physically active and healthy by recommending and tracking their daily exercise routines.

Chat bots can provide professional guidance and mentorship to each employee. Investing in the career growth of each employee is perhaps the highest ROI effort for HR teams and business organizations. Chat bots can develop customized learning and development plans for each individual. This can include both soft and hard skills that are required for professional success. Chat bots can reinforce the culture of the organization by recommending appropriate developmental courses. Chat bots can assess employee skills and recommend courses and modules that employees can subscribe to. Chat bots can also connect them to mentors within the organization.

Of course, there are important issues around privacy and confidentiality that must be addressed. However HR conversations, by their very nature, tend to involve sensitive and personal information. These issues can be suitably addressed by providing users and organizations adequate control over their data.

Essentially, the chat bots can be an always-on, highly engaged, extremely personalized companion - the perfect HR Manager. HR teams that adopt chat bots will find themselves getting closer to successfully accomplishing the many impossible tasks and conflicting goals that their organizations place on them.

\section{Corresponding author}

Beerud Sheth can be contacted at: beerud@gupshup.io 